\title{
Frequencies of HLA-DQ2 and HLA-DQ8 haplotypes in Czech and Slovak coeliac patients and the healthy population
}

\author{
Katerina Wroblova', Michal Kolorz', Igor Pav², Zuzana Horakova', Petra Filipova', Milan Bartos³ \\ and Ladislava Bartosova1
}

'Department of Human Pharmacology and Toxicology, UVPS Brno, Brno, Czech Republic; ${ }^{2}$ Gastroenterological Clinic SMU and University Hospital in Bratislava-Petrzalka, Bratislava, Slovak Republic; ${ }^{3}$ Department of Natural Drugs, UVPS Brno, Brno, Czech Republic

\begin{abstract}
Coeliac disease is an autoimmune disorder with genetic predisposition. The aim was to determine the frequency of HLA-DQ2 and HLA-DQ8 in Czech and Slovak patients and the healthy population. The study included 127 patients and 66 healthy volunteers. HLA-DQ2 was identified in $85.03 \%$ patients, and $24.24 \%$ healthy individuals $(\mathrm{P}=0.0001 ; \quad$ OR17.7632; $\mathrm{Cl}=8.4347-37.4088)$. HLA-DQ8 was identified in $11.81 \%$ patients and $15.5 \%$ healthy individuals. HLA-DQ8 occurred more often in HLA-DQ2negative patients compared to HLA-DQ2-positive patients $(P=0.0494 ;$ OR3.5; $\mathrm{Cl} 1.0428-11.7468)$. At least one of the studied HLA-variants was found more often in patients than in healthy individuals $(P=0.0001 ; 0 R 58.8 ; \mathrm{Cl}$ 7.6856-449.8602).
\end{abstract}

Key words: coeliac disease, HLA haplotype, HLA-DQ2, HLA-DQ8, Czech and Slovak population

Received: 07 November, 2013; revised: 06 March, 2014; accepted: 07 March, 2014; available on-line: 22 March, 2014

\section{INTRODUCTION}

Coeliac disease (CD) is a common autoimmune disorder. Untreated CD can lead to the development of serious complications and it is estimated that only 10-15\% of the affected persons are correctly diagnosed, treated and monitored (NICE 2009). Due to this, timely diagnosis plays a key role. $\mathrm{CD}$ has been associated with the haplotype HLA-DQ2 (alleles DQA1*05/DQB1*02) and HLA-DQ8 (alleles DQA1*03/DQB1*0302)(Sollid et al., 1989; Karell et al., 2003). In 2012, the European Society for Pediatric Gastroenterology, Hepatology, and Nutrition recommended genetic examination of risk haplotypes HLA-DQ2 and HLA-DQ8 as possible method for diagnosis (Husby et al., 2012), since the absence of these haplotypes makes CD unlikely (Megiorni et al., 2009).

The aim of this study was to determine the frequencies of haplotypes HLA-DQ2 and HLA-DQ8 in the Czech and Slovak population and compare the occurrence of these haplotypes between patients and the healthy population.

\section{MATERIALS AND METHODS}

The study included 127 patients with CD and 66 healthy volunteers without CD (who did not have any associated autoimmune disorder) and without familial occurrence of this disease. Patients included in the study have been monitored at specialised workplaces in the Czech and Slovak Republics. All volunteers accepted into the study confirmed their consent for genetic testing by signing the Informed Consent and by filling in the Questionnaire. The study was approved by the Ethics Committee of the Merciful Brothers Hospital in Brno (Czech Republic).

We optimised and verified an allele-specific multiplexPCR method for determination of the risk haplotypes. Specificity of primer sequences allows the formation of a PCR product only when a disease predisposing allele is present in the genomic DNA. The consequent optimisation of the reaction mixture and conditions allowed us to carry out allele-specific PCR in the multiplex system, where it is possible to detect several alleles at the same time. Primers for amplification of parts of the $\mathrm{TNF} \alpha$ gene were added to the mixture as an internal control to avoid false negative results in case when the patient doesn't carry any of the monitored alleles. Genotyping was performed on two parallel PCR reactions where one PCR reaction detected the haplotype HLA-DQ2 (alleles $\mathrm{DQA} 1{ }^{*} 05$ and $\left.\mathrm{DQB} 1{ }^{*} 02\right)$ and the second the haplotype HLA-DQ8 (alleles DQA1*03 and DQB1*0302). Results of the proposed methodologies were validated by DNA sequencing and internal positive and negative controls. The products were separated by electrophoresis in a $2.5 \%$ agarose gel. Visualization was performed on a UV transilluminator by $312 \mathrm{~nm}$ light.

\section{Interpreting of results}

Individuals, in whom both alleles DQA $1 * 05$ and DQB1*02 were identified were marked HLA-DQ2positive. Carriers of both alleles, i.e. DQA $1 * 03$ and DQB1*0302 were marked HLA-DQ8-positive.

\section{Statistical analysis}

Results were interpreted by statistical analysis using the Fisher's exact test, odds ratio and confidence interval calculations. Values of $P<0.05$ were considered significant. Disease risks are expressed as $1: N$, where $N$ is the number of individuals among whom one patient is present. Considering a disease prevalence of 1:100 in the general population, for each HLA-DQ category, N is calculated as a percentage of controls with that particular HLA-DQ status multiplied by 100 and divided

e-mail: kolorzm@vfu.cz

Abbreviations: CD coeliac disease; HLA human leukocyte antigen; $\mathrm{PCR}$ polymerase chain reaction; TNFa tumor necrosis factor alpha 
Table 1. Frequency of the studied HLA-variants (HLA-DQ2, HLADQ8)

None: absence of all studied HLA-variants (HLA-DQ2, HLA-DQ8, DQA $1{ }^{*} 05$ and $\left.\mathrm{DQB} 1{ }^{*} 02\right) ;{ }^{*} P<0.01,{ }^{*} P<0.05$

\begin{tabular}{|c|c|c|c|c|c|}
\hline & $\mathrm{n}$ & & HLA-DQ2 & HLA-DQ8 & None \\
\hline \multirow[t]{2}{*}{ Patients } & 127 & Cases & $108^{* *}$ & 15 & $1^{* *}$ \\
\hline & & (\%) & 85.03 & 11.81 & 0.8 \\
\hline \multirow[t]{2}{*}{ Controls } & 66 & Cases & 16 & 9 & 21 \\
\hline & & (\%) & 24.24 & 15.5 & 31.8 \\
\hline
\end{tabular}

by the percentage of patients with the same DQ typing (Megiorni et al., 2009).

\section{RESULTS}

Haplotype HLA-DQ2 was identified in $85.03 \%$ patients, and $24.24 \%$ healthy individuals. Haplotype HLADQ8 was identified in $11.81 \%$ patients and $15.5 \%$ healthy individuals (Table 1). Statistical analysis proved significant differences in the occurrence of haplotype HLA-DQ2 between patients with CD and healthy individuals $(P=0.0001 ;$ OR17.7632; $\mathrm{CI}=8.4347-37.4088)$. No significant difference in the presence of haplotype HLADQ8 between the groups was found.

When the presence of haplotype HLA-DQ8 was studied in correlation with the presence of haplotype HLADQ2, it was found that haplotype HLA-DQ8 occurred significantly more often in HLA-DQ2-negative patients compared to HLA-DQ2-positive patients $(P=0.0494$; OR3.5; CI 1.0428-11.7468). At least one of the studied HLA-variants (HLA-DQ2, HLA-DQ8 or HLADQ2 heterodimer chain, coded by allele DQB1*02 or DQA $1 * 05)$ was found significantly more often in patients than in healthy individuals $(P=0.0001$; OR58.8; CI 7.6856-449.8602). Only one patient was not a carrier of haplotype HLA-DQ2 and HLA-DQ8 and of neither of the alleles DQA $1 * 05$ and DQB1*02.

\section{DISCUSSION}

Coeliac disease is a widespread autoimmune disorder; however, only a part of patients is correctly diagnosed due to an altered disease phenotype. Standard gastrointestinal symptoms (diarrhoea, constipation, vomiting, flatulence, weight loss, etc.) occur in a smaller percentage of patients and are mainly characteristic in children. Symptoms, which have non-specific extraintestinal character, are more usual. The most common are iron-deficiency anaemia, reduced bone mineral density, dermatitis herpetiformis, chronic fatigue, depression, amenorrhoea, etc. (Hill et al., 2005; NICE 2009; Husby et al., 2012). Unfortunately, these antypical symptoms are not always correctly diagnosed as coeliac disease. Untreated disease can result in the development of serious complications or even fatal outcome. It is therefore important to identify such individuals on time and start a gluten-free diet.

In the course of the study, we succeeded in designing and optimising of a method for the determination of risk CD haplotypes HLA-DQ2.5 and HLA-DQ8. In the Czech and Slovak population the haplotype HLA-DQ2 occurs in $85.03 \%$ of the patients and $24.24 \%$ of healthy individuals. These results are comparable with the occurrence of HLA-DQ2.5 Italians (80.8-86\% in patients, $21.8 \%$ in controls),s French
(87\% in patients) and English (87.8\% in patients). In Scandinavia, the frequency is slightly higher (92-96\% in patients) (Karell et al., 2003; Margaritte-Jeannin et al., 2004; Megiorni et al., 2008).

We confirmed a highly significant association of Haplotype HLA-DQ2 with coeliac disease, and our findings are in agreement with results of other studies (Sollid et al., 1989; Karell et al., 2003; Megiorni et al., 2009; Piccini et al., 2012). Patients with coeliac disease have this allelic combination 17 to 18 times more often than healthy individuals which may suggest that they contribute to the development of CD. No significant difference was found in the frequency of haplotype HLA-DQ8 between individual genotyped groups; this haplotype is evenly spread in all groups. Similar results were obtained for the Italian population (Megiorni et al., 2009; Piccini et al., 2012). Nevertheless, when we compared patients, who carry haplotype HLA-DQ2, with patients without this haplotype, HLA-DQ8 occurred significantly more often in HLA-DQ2-negative than in HLA-DQ2-positive patients. HLA-DQ2-negative patients with coeliac disease carried haplotype HLA-DQ8 3-4 times more often compared to HLADQ2-positive patients. These findings point to a probable conclusion that if a patient with coeliac disease does not have haplotype HLA-DQ2, they more often have at least haplotype HLA-DQ8. The whole group of patients included only one, who was not a carrier of haplotype HLA-DQ2 or HLA-DQ8 and of neither of the alleles DQA1*05 and DQB1*02. This implies that absence of all these genetic variants rules out the risk of the CD development with almost 100\% certainty. Similar results were reported by Karell et al. (2003) and Polvi et al. (1998), who recommended, based on their experimental results, to monitor not only haplotypes HLA-DQ2 and HLA-DQ8 as predisposing factors for the development of coeliac disease, but also the presence of individual HLA-DQ2 heterodimer chains (DQA $1 * 05$ and $\mathrm{DQB} 1 * 02)$.

Based on the risk calculation, the development of CD seems improbable $(1: 4025)$ in patients, who do not carry any of the haplotypes HLA-DQ2 or HLA-DQ8, or any of the alleles coding the heterodimer HLADQ2 (DQA1*05 and DQB1*02). These results support the results of recent studies by Piccini et al. (2012) and Megiorni et al. (2009).

Genetic examination is useful when the diagnosis is not definite. This usually happens when findings from intestinal mucosa are ambiguous, serum antibodies negative and when the patient had started a gluten-free diet before the diagnosis was confirmed. Furthermore, genetic screening is a simple and non-invasive method, suitable to eliminate association between a certain clinical problem (anaemia, osteoporosis, neurological problems, infertility, etc.) with coeliac disease. In all these cases, the absence of the above listed HLA molecules and individual alleles rules out the coeliac disease, with the exception of a clear clinical picture confirming the presence of CD.

In conclusion, we assessed the frequencies of haplotypes HLA-DQ2 and HLA-DQ8 in Czech and Slovak patients and healthy population. HLA-DQ2 is associated with CD. In HLA-DQ2-negative patients, there is more often present at least the haplotype HLA-DQ8. The presence of HLA molecules does not confirm this diagnosis absolutely; it only indicates the presence of a genetic predisposition. However, the absence of all the monitored alleles makes the onset of CD unlikely. 


\section{Conflict of interest}

"The authors have declared that no competing interest exists"

\section{Acknowledgements}

The authors would like to express their thanks for the cooperation to the Coeliacs Society Brno.

The authors would like to express their thanks for the financial support to the Internal Grant Agency of the University of Veterinary and Pharmaceutical Sciences Brno IGA VFU 91/2012/FaF.

\section{REFERENCES}

Hill ID, Dirks MH, Liptak GS, Colletti RB, Fasano A, Guandalini S, Hoffenberg EJ, Horvath K, Murray JA, Pivor M, Seidman EG (2005) Guideline for the diagnosis and treatment of celiac disease in children: recommendations of the North American Society for Pediatric Gastroenterology, Hepatology and Nutrition. J Pediatr Gastroenterol Nutr 40: 1-19.

Husby S, Koletzko S, Korponay-Szabó IR, Mearin ML, Phillips A, Shamir R, Troncone R, Giersiepen K, Branski D, Catassi C, Lelgeman M, Mäki M, Ribes-Koninckx C, Ventura A, Zimmer KP (2012) European Society for Pediatric Gastroenterology, Hepatology and Nutrition guidelines for the diagnosis of coeliac disease. I Pediatric Gastroenterol Nutr 54: 136-160.
Karell K, Louka AS, Moodie SJ, Ascher H, Clot F, Greco L, Ciclitira PJ, Sollid LM, Partanen J (2003) HLA types in celiac disease patients not carrying the DQA1*05-DQB1*02 (DQ2) heterodimer: results from the European Genetics Cluster on Celiac Disease. Hum Immunol. 64: 469-477.

Margaritte-Jeannin P, Babron MC, Bourgey M, Louka AS, Clot F, Percopo S, Coto I, Hugot JP, Ascher H, Sollid LM, Greco L, Clerget-Darpoux F (2004) HLA-DQ relative risks for coeliac disease in European populations: a study of the European Genetics Cluster on Coeliac Disease. Tissue Antigens 63: 562-567.

Megiorni F, Mora B, Bonamico M, Barbato M, Montuori M, Viola F, Trabace S, Mazzilli MC (2008) HLA-DQ and susceptibility to celiac disease: evidence for gender differences and parent-of-origin effects. Am J Gastroenterol 103: 997-1003.

Megiorni F, Mora B, Bonamico M, Barbato M, Nenna R, Maiella G, Lulli P, Mazzilli MC (2009) HLA-DQ and risk gradient for celiac disease. Human Immunology 70: 55-59.

NICE clinical guideline 86 (2009) Coeliac Disease-Recognition and Assessment of Coeliac disease. National Institute for Health and Clinical Excellence. 4-86.

Piccini B, Vascotto M, Serracca L, Luddi A, Margollicci MA, Balestri P, Vindigni C, Bassotti G, Villanacci V (2012) HLA-DQ typing in the diagnostic algorithm of celiac disease. Rev Esp Enferm Dig 104: 248-254.

Polvi A, Arranz E, Fernandez-Arquero M, Collin P, Mäki M, Sanz A, Calvo C, Maluenda C, Westman P, de la Concha EG, Partanen J (1998) HLA-DQ2-negative celiac disease in Finland and Spain. Hum Immunol 59: 169-175. 